\title{
Sentinel lymph node biopsy with one-step nucleic acid assay relegates the need for preoperative ultrasound-guided biopsy staging of the axilla in patients with early stage breast cancer
}

\author{
BELLO INUA $^{1}$, VICTORIA FUNG ${ }^{1}$, NOUR AL-SHURBASI ${ }^{1}$, SARAH HOWELLS ${ }^{2}$, OLGA HATSIOPOULOU ${ }^{2}$, \\ PRAVEEN SOMARAJAN ${ }^{2}$, GREGORY J. ZARDIN ${ }^{3}$, NORMAN R. WILLIAMS ${ }^{4}$ and STAN KOHLHARDT $^{1}$ \\ Departments of ${ }^{1}$ Breast, Plastic and Reconstructive Surgery; ${ }^{2}$ Breast Screening and Breast Imaging and \\ ${ }^{3}$ Histopathology, Royal Hallamshire Hospital, Sheffield S10 2JF; ${ }^{4}$ Surgical and Interventional Trials Unit, Division of \\ Surgery and Interventional Science, Faculty of Medical Sciences, University College London, London W1W 7JN, UK
}

Received October 20, 2019; Accepted August 21, 2020

DOI: $10.3892 / \mathrm{mco} .2021 .2213$

\begin{abstract}
Avoiding axillary node clearance in patients with early stage breast cancer and low-burden node-positive axillary disease is an emerging practice. Informing the decision to adopt axillary conservation is examined by comparing routine preoperative axillary staging using ultrasound (AUS) \pm AUS biopsy (AUSB) with intraoperative staging using sentinel lymph node biopsy (SLNB) and a one-step nucleic acid cytokeratin-19 amplification assay (OSNA). A single-centre, retrospective cohort study of 1,315 consecutive new diagnoses of breast cancer in 1,306 patients was undertaken in the present study. An AUS \pm AUSB was performed on all patients as part of their initial assessment. Patients who had a normal ultrasound (AUS-) or negative biopsy (AUSB-) followed by SLNB with OSNA \pm axillary lymph node dissection (ALND), and those with a positive AUSB (AUSB+), were assessed. Tests for association were determined using a $\chi^{2}$ and Fisher's Exact test. A total of 266 (20.4\%) patients with cT1-3 cN0 staging received 271 AUSBs. Of these, 205 biopsies were positive and 66 were negative. The 684 patients with an AUS-/AUSB-assessment proceeded to SLNB with OSNA. AUS sensitivity and negative predictive value (NPV) were 0.53 [0.44-0.62; $95 \%$ confidence interval $(\mathrm{CI})]$ and $0.58(0.53-0.64,95 \% \mathrm{CI})$, respectively. Using a total tumour load cut-off of 15,000 copies $/ \mu 1$ to predict $\geq 2$ macro-metastases, the sensitivity and NPV for OSNA were $0.82(0.71-0.92,95 \% \mathrm{CI})$ and $0.98(0.97-0.99,95 \% \mathrm{CI})$ (OSNA vs. AUS P $<0.0001$ ). Of the AUSB+ patients, $51 \%$ had $\leq 2$ positive nodes following ALND and were potentially
\end{abstract}

Correspondence to: Dr Stan Kohlhardt, Department of Breast, Plastic and Reconstructive Surgery, Royal Hallamshire Hospital, 4 Claremont Place, Sheffield S10 2JF, UK

E-mail: stan.kohlhardt@nhs.net

Key words: axillary ultrasound, axillary conservation, one step nucleic acid amplification assay, cytokeratin-19, breast cancer, sentinel lymph node over-treated. Where available, SLNB with OSNA should replace AUSB for axillary assessment in cT1-2 cN0 patients with $\leq 2$ indeterminate nodes seen on AUS.

\section{Introduction}

Patients with a new diagnosis of early breast cancer require axillary node staging to quantify regional metastatic involvement, and this provides key prognostic information used for determination of individual treatment plans. Staging of the axilla prior to surgery using ultrasound \pm axillary ultrasound (AUS)-guided biopsy (AUSB) and routine fixation with haematoxylin and eosin (H\&E) staining, is frequently performed to obtain detailed information in patients newly diagnosed with breast cancer $(1,2)$. The removal of all axillary nodes with an axillary lymph node dissection (ALND) is recommended in patients with a positive biopsy. When the biopsy is negative, operative assessment is required with a sentinel lymph node (SLN) biopsy (SLNB) (1). When the SLNB is found to be free from metastatic disease, no further axillary treatment is recommended.

Patients with early stage (T1-2) disease, $\leq 2$ macrometastatic SLNs who are treated with SLNB alone, breast conservation surgery, whole-breast radiotherapy and adjuvant systemic therapy exhibit similar disease-free and overall survival rates compared with those who undergo $\operatorname{ALND}(3,4)$. As such, ALND may be considered unnecessary or in excess for treatment of patients with early breast cancer and a low burden of axillary node involvement. A routine decision to proceed with ALND following the return of a single positive biopsy from AUSB may thus be out of step with emerging and contemporary practice.

A reliable intraoperative technique that identifies, quantifies and predicts axillary lymph node involvement may offer selective and more conservative surgical treatment of the axilla in a single procedure. ALND, and its associated increased risk of morbidity, may be avoided whilst also providing benefits to these patients, conserving resources and remaining in compliance with emerging clinical practice guidelines $(3,5)$. SLNB combined with an intraoperative molecular-based 
assessment, one-step nucleic acid cytokeratin-19 (CK-19) amplification assay (OSNA), provides an objective whole-node assessment of SLN disease burden that is independent of the size or number of lymph nodes tested (6). For these reasons, OSNA possesses greater potential for predicting residual axillary nodal involvement compared with routine histopathological assessment, as histopathological assessment does not offer timely intraoperative SLN evaluation, is subjective, categorical and is at increased risk of sampling errors with sub-total node assessment (7,8). OSNA amplifies CK19 mRNA in SLN samples, typically providing a quantitative measurement of metastatic disease burden within $35 \mathrm{~min}$. OSNA can be used to inform prognosis and stratify nodal disease burden into negative, micro-metastatic, $\leq 2$ node macro-metastatic, and $>2$ and $>4$ node macro-metastatic levels (7-11). The total CK19 mRNA copy number of a SLNB [(total tumour load (TTL)] may predict non-SLN (NSLN) involvement and axillary node disease burden, facilitating the decision to proceed with, or avoid complete ALND.

OSNA is currently used in $>300$ centres across Europe and in Japan (Sysmex Europe $\mathrm{GmbH}$ ). It has been fully validated and was approved for routine clinical use in the UK by the National Institute of Clinical Excellence (NICE) in 2013 (12). In routine operating practices, SLNB is performed first and the node(s) are sent to the pathology department for OSNA. Whilst awaiting the results, the surgeon will continue with the primary breast procedure and, when appropriate, closure of the primary breast wound. However, $70 \%$ of OSNA procedures are negative. The latest analyser can return results within 35 min so there is essentially no delay introduced by the procedure. The cost-effective savings and improvements in the patient care are provided by proceeding to perform a single procedure for the $\sim 30 \%$ of patients who actually require ALND. There is no need for the patient to return to hospital for ALND. There is no duplication of theatre time, anaesthetic or surgical resources (including time, reusables, disposables and surgical instrument re-sterilization). There is no inconvenience to the patient with a return to hospital, and operating theatre availability is increased downstream for other patients. NICE concluded that the RD-100i OSNA system was likely to be a cost-effective use of resources, equally or more cost effective than postoperative histopathology (12). Fiscal savings in terms of reduced secondary surgeries and bed occupancy (in 2015) were potentially worth $€ 150$ per patient (12-14).

The aim of the present study was to compare the accuracy and utility of SLNB with OSNA to AUSB with routine histochemistry in staging the axilla of patients with a new diagnosis of early breast cancer. Using a retrospective cohort study of consecutive patients over a 3-year period, an extended role for OSNA to define a group of patients for whom pre-operative assessment of nodal staging with AUSB is redundant and may represent an unnecessary physical intervention with duplication of pathology resources was identified.

\section{Patients and methods}

Patients. The present study was a retrospective, single-centre cohort study of consecutive patients, treated between December 2012 and August 2015, with primary invasive cT1-3 breast carcinoma. All patients received AUS \pm AUSB, and the patients were divided into two main groups: i) Patients with a single positive AUSB (AUSB+) who proceeded to ALND (median age, 61.2 years; age range 23-93; 191 female, 0 male) and ii) patients with a normal AUS or negative biopsy (AUSB-) followed by SLNB with OSNA \pm ALND (median age, 54.2 years; age range, 23-92; 687 female, 4 male).

Patient data were anonymized and collected retrospectively, without influence on patient therapy. The present study was approved by the Clinical Effectiveness Unit of Sheffield Teaching Hospitals NHS Trust. As part of a service evaluation of two standardised diagnostic interventions, there was no requirement to obtain informed consent from the patients. No patient was interviewed or surveyed, and no identifiable patient data were used in the study. Therefore, consent for participation from patients was waived.

Patients who received neo-adjuvant chemotherapy prior to AUS \pm AUSB, underwent ipsilateral axillary surgery, or had recurrent disease or extensive ductal carcinoma in situ (DCIS) without invasion were excluded. Bilateral breast cancer cases were treated as separate entities. A threshold of 1-2 metastatic SLNs was used as a surrogate measure of low-burden axillary node disease. This threshold corresponds to the level reported in the American College of Surgeons Oncology Group (Alliance) ACOSOG Z0011 trial $(3,4)$. Analysis was performed on the whole patient group and a subset of patients, separately identified from the whole group, who met the full criteria of the ACOSOG Z0011 trial; that is, no pre-operative chemotherapy, T1 or T2 tumour, 1 or 2 positive SLN's, breast-conserving surgery and planned whole breast radiotherapy. ACOSOG trial selection criteria compliant patient characteristics are not detailed since patient outcomes (such as local recurrence, disease-free or overall survival) are not measured or relevant, and trial outcomes comparison unintended. In contrast with the ACOSOG Z0011 study, all patients in the present study underwent AUS prior to breast surgery. Those with an abnormal lymph node morphology underwent immediate ultrasound guided biopsy, and those with confirmed metastatic disease were recommended to undergo ALND without SLNB \pm OSNA, the protocol used at the time of this study.

Pre-operative assessment of the axilla. B mode ultrasound examination of the axilla was performed by experienced breast radiologists and advanced breast practitioners, following a standardised protocol (15). With the patient placed in a supine position and the ipsilateral hand resting behind the head, the axilla was scanned in a longitudinal (L) and transverse (T) direction using a Siemens Acuson S2000 18L6 high-density high frequency linear-array transducer (5.5-18.0 MHz; Siemens AG). Lymph nodes with an abnormal appearance were identified using qualitative criteria including size, oval or round shape, absent fatty hilum, abnormal peripheral blood flow, sharpness of the margin and focal thickening of the cortex (16-18). These nodes were targeted and the $\mathrm{L} / \mathrm{T}$ axis ratio (Solbiati index), hilum and cortical thickness were measured. If there was $>1$ abnormal node in the axilla, the most morphologically abnormal node was sampled. Lymph nodes with an absent fatty hilum, L/T index $<2$ and/or cortical thickness $>2.9 \mathrm{~mm}$ were biopsied, under direct ultrasound guidance, using local anaesthetic and a $90 \mathrm{~mm} 18 \mathrm{~g}$ core-cut needle with 
automated throw (Achieve programmable automatic biopsy system; CareFusion).

SLN identification and OSNA. SLNB was performed using a standard protocol using a combination of radiopharmaceutical and blue dye (19). ${ }^{99 \mathrm{~m}} \mathrm{Tc}-$ labelled albumin nanocolloid (Nanocoll ${ }^{\circledR}$; GE Healthcare) was injected intradermally $(0.1-0.5 \mathrm{ml})$ at a single periareolar site corresponding to the tumour quadrant; $40 \mathrm{MBq}$ the day before surgery or $20 \mathrm{MBq}$ on the day of surgery. Patent Blue V Dye (Laboratoire Guebert; $2 \mathrm{ml}$ undiluted) was injected subdermally at a single periareolar site corresponding to the tumour quadrant immediately prior to surgery. Under general anaesthetic, SLNs were identified and removed prior to breast tumour excision, and sent on ice to the Pathology Department; no more than 2 nodes were sent for assessment by OSNA. Any additional SLNs were sent for routine fixation, H\&E staining and delayed reporting (20). Therapeutic local excision, therapeutic mammoplasty or mastectomy was performed as part of the planned breast cancer treatment. Each SLN, trimmed of fat, was weighed and recorded. SLNs weighing $<50 \mathrm{mg}$ were too small to be processed by OSNA, and therefore used for routine histological assessment. SLNs weighing $>600 \mathrm{mg}$ were divided into two or more pieces and processed separately, and the results combined. The OSNA assay was performed according to the manufacturer's protocol (Sysmex Europe $\mathrm{GmbH}$ ) (6). Each SLN was homogenized in $4 \mathrm{ml}$ homogenizing buffer on ice. The lysate was centrifuged to remove fat, cellular debris and other contaminants, and the mRNA containing supernatant was extracted and diluted. A $2 \mu 1$ aliquot of the buffered lymph node lysate was used for automated quantitative amplification of CK19 mRNA via reverse transcription loop-mediated isothermal amplification (RT-LAMP) using a ready-to use reagent kit on a RD-100i system (Sysmex Europe GmbH). The rate of amplification was measured spectrophotometrically and the CK19 copy number calculated by comparison to a standard curve. Based on the number of CK19 mRNA copies $/ \mu 1$, the result was assessed in accordance with the cut-off levels determined by Tsujimoto et al (6) with non-metastatic as $<250$ copies $/ \mu 1$, micro-metastasis as 250-5,000 copies $/ \mu 1$ and macro-metastasis defined as $>5,000$ copies/ $\mu 1$ of CK19 mRNA. The OSNA results were communicated by telephone to the surgeon within 45 min of sample receipt. Patients with at least one macro-metastasis on intraoperative OSNA analysis underwent levels I, II and III ALND. Between December 2012 and June 2013, a positive OSNA result for one or two nodes with micro-metastases meant the patient immediately underwent ALND. In June 2013, the departmental protocol was amended to recommend the removal of two further nodes for routine histological processing with a delayed ALND if these returned macro-metastatic involvement. All remaining lymph nodes not used for OSNA were processed according to the UK Breast Cancer pathology protocol (20). Lymph nodes $<5 \mathrm{~mm}$ were bisected whereas larger nodes were sectioned into $3 \mathrm{~mm}$ single sections and assessed using H\&E staining.

Statistical analysis. For statistical analysis, when $\geq 2$ SLNs were involved, the combined value of CK19 mRNA copies was calculated. The TTL was defined as total CK19 mRNA copy number in all the positive SLNs (copies/ $\mu$ l). The TTL of the macro-metastatic SLNB sample was compared with the total lymph node status and NSLN status of ALND, following routine histological assessment with H\&E staining. For subset data interpretation, the TTL was set at 5,000 copies/ $\mu 1$ for recommendation of ALND and 15,000 copies/ $\mu 1$ as a threshold of 2 positive nodes (10). Tests for association were determined using a $\chi^{2}$ test or Fisher's Exact test between the AUSB group and the SLNB/ALND group. All tests were two-tailed and $\mathrm{P}<0.05$ was considered to indicate a statistically significant difference.

\section{Results}

Between December 2012 and August 2015, there were 1,315 new diagnoses of cT1-3 carcinoma in 1,306 consecutive patients. Of these, 266 (20.4\%) patients with cT1-3 cN0 staging received 271 AUSBs as part of their assessment; 205 AUSB+ and 66 AUSB-. Based on the results, 23 cases (14 AUSB+ and 9 AUSB-) were excluded from the 271 AUSB analyses; 9 patients were treated with primary endocrine therapy only, 8 patients had DCIS only on subsequent pathological reporting, 3 patients had metastatic disease on presentation, 2 patients had recurrent disease and 1 patient had a contralateral lymph node biopsy diagnosing intercurrent chronic lymphocytic leukaemia. Complete AUSB assessment was performed on 191 AUSB+ and 57 AUSB-biopsies.

A total of 700 SLNB with OSNA procedures were performed in 691 patients with an AUS-/AUSB-assessment. A total of 16 cases were excluded from this group; 15 patients had extensive DCIS only on the final histological analysis, and 1 patient had received incomplete neo-adjuvant therapy (Fig. 1). The remaining 349/1,306 patients had normal AUS assessments and did not proceed to SLNB with OSNA for multiple reasons including advanced age and/or concurrent illness (unfit for surgery), metastatic disease at presentation, previous ipsilateral axillary surgery or a decision to provide neo-adjuvant chemotherapy or primary endocrine therapy. Macro-metastatic lymph node involvement was finally diagnosed in 348/971 of the axillae studied (35.8\%); 279 of these 348 axillae were further characterised as $>2$ or $\leq 2$ macro-metastatic disease burden by ALND. The remaining 69/348 were lost to treatment naïve ALND analysis by above and below mentioned exclusions, including standard pre-ALND treatment means.

Of the 191 AUSB+ assessments, 137 proceeded directly to ALND; 54 were censored after treatments that precluded further analysis of nodal involvement (31 received chemotherapy, 19 received primary endocrine therapy only, $3 \mathrm{had}$ breast only surgery for local control and 1 received radiotherapy only). A total of 70/137 (51\%) of the AUSB+ patients who subsequently underwent ALND had $\leq 2$ positive nodes. For an AUSB+, estimating a nodal burden of $>2$ (positive) axillary lymph node metastases was expressed as the sensitivity and negative predictive value (NPV) of AUS of 0.53 [(0.44-0.62), 95\% confidence interval (CI)] and 0.58 (0.53-0.64, $95 \% \mathrm{CI}$ ), respectively (Table I). The false negative rate (FNR) of AUSB correctly predicting an axillary node burden of $>2$ macro-metastatic nodes was $47 \%$ (accuracy, 53.8\%).

The sensitivity and NPV for SLNB + OSNA, using a TTL cut-off of 15,000 copies/ $\mu 1$ for predicting > 2 macro-metastases, were $0.82(0.71-0.92,95 \% \mathrm{CI})$ and $0.98(0.97-0.99,95 \% \mathrm{CI}$; 


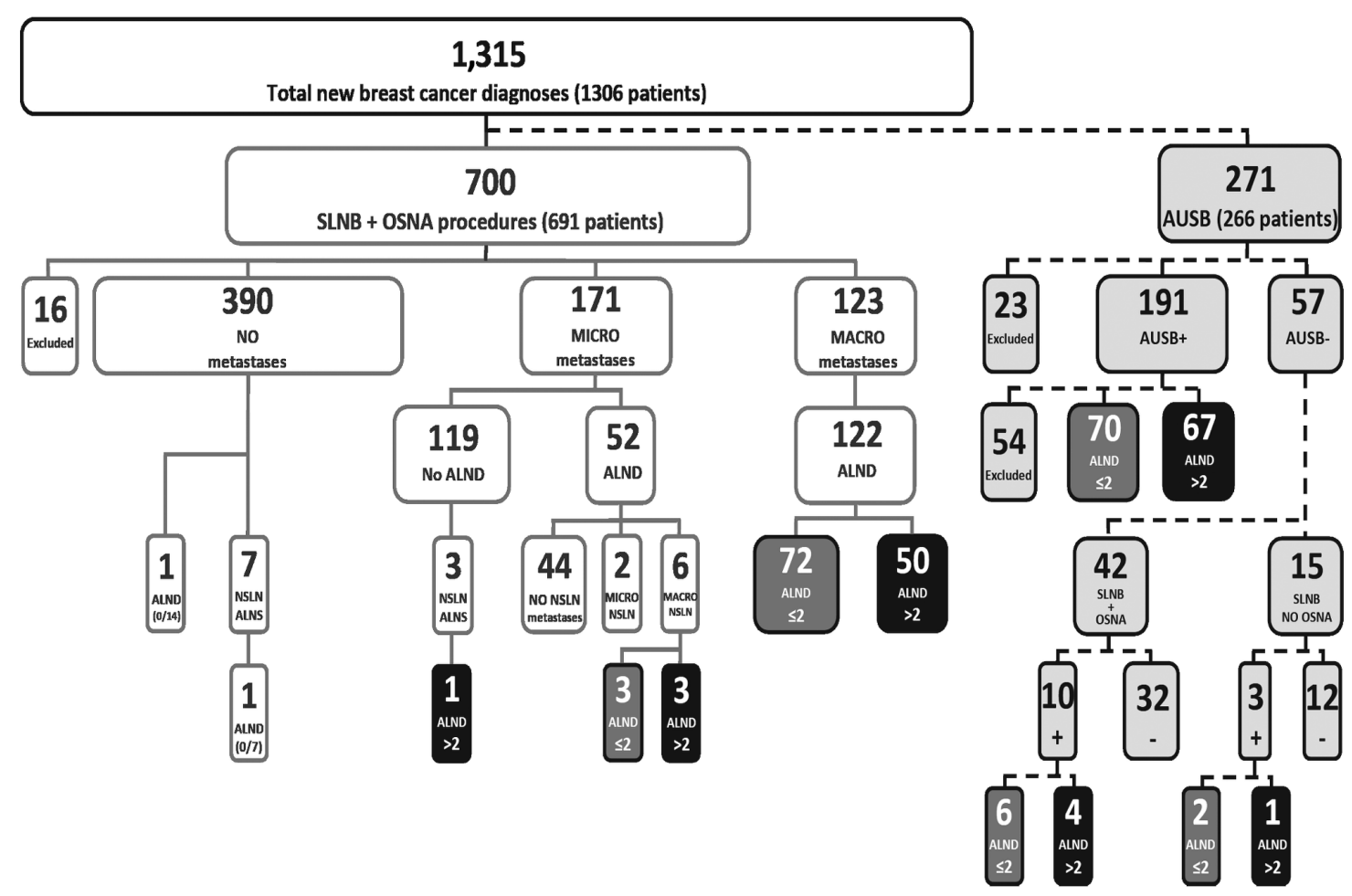

Figure 1. Cohort distribution flow chart. SLNB, sentinel lymph node biopsy; OSNA, One-step Nucleic Acid Amplification; ALND, axillary lymph node dissection; NSLN, non-sentinel lymph node; AUSB, axillary ultrasound biopsy; +, positive node (metastatic disease present); -, negative node (metastatic disease absent); MICRO, micro-metastases; MACRO, macro-metastases; ALNS, axillary lymph node sample.

$\mathrm{P}<0.0001$, OSNA vs. AUS) for all patients (Table II) and 0.87 (0.73-1.0) and 0.99 (0.99-1.0; $\mathrm{P}<0.0001$, OSNA vs. AUS) for patients matched to the ACOSOG Z0011 trial inclusion criteria, identified and analysed separately (Table III). The FNR of SLNB + OSNA correctly predicting an axillary node burden of $>2$ macro-metastatic nodes was $18 \%$ (accuracy, $87.6 \%$ ) in the whole group and 13\% (accuracy, 90.8\%) for the Z0011 matched group. The NPV for all axillae not associated with breast conserving surgery was 0.949 (95\% CI, 0.906-0.973).

\section{Discussion}

The clinical utility of AUS \pm AUSB to stage clinically node-negative early breast cancer is contested. The ACOSOG Z0011 trial, and several other studies have reported that patients with low burden axillary node involvement do not require ALND, demonstrating no adverse impact on locoregional control or overall survival $(3,4,21-23)$. These findings are resulting in surgical practice moving towards selective conservation of the node involved axilla $(4,5,24,25)$. In the present study, $51 \%$ of patients with AUSB+ had $\leq 2$ involved nodes following ALND. This observation is consistent with the findings of other studies, which reported between $41-52 \%$ of cases (26-30) and supports the notion of excess treatment in a substantial proportion of patients. As an instrument for deciding between ALND and axillary conservation, AUSB+ alone does not provide sufficient discrimination, particularly in Z0011 criteria compliant patients. Meeting the demands of emerging clinical practices to confidently deliver conservative management of the low-burden node-positive axilla in early breast cancer requires scales of quantification and stratification of axillary nodal disease burden greater than that provided by AUS \pm AUSB (31).

The sensitivity of AUS assessment is highly dependent on the prevalence and extent of axillary tumour burden (26,32-35). Stachs et al (15) reported AUS sensitivity of $45.2 \%$, similar to the present study. With regards to avoiding unnecessary ALND surgery and its associated complications, the sensitivity and specificity of AUSB calculated in the present study questions the logic of performing the procedure when only one node, or possibly two or have appearances considered indeterminate on imaging in this group of patients. These findings suggest that AUSB should not be performed routinely in patients with a new presentation of $\mathrm{cT} 1-2 \mathrm{cN} 0$ breast cancer, but used selectively when the ultrasound analysis identifies multiple (>2) node involved, in disagreement with the clinical findings.

Nodal macro-metastases were present in 35.8\% (348/971) of the present cohorts' axillae. Just over half of these (59\%; 205/348) were detected, but not quantified, by AUS assessment. On the other hand, a negative AUS assessment did not exclude the possibility of lymph node metastases. Of the AUSBs, 66/271 (24.3\%) were negative, of which 13/57 (22.8\%) AUSB-axillae and 130/700 (18.6\%) AUS-axillae had macro-metastatic nodal disease following ALND. The SLNB FNR is inversely related to the number of nodes removed (36), supporting the observation of a high FNR for AUSB with sub-total, core-cut biopsy of a single node. In the present study, the observed mean yield of 1.94 SLNs for OSNA TTL per patient, is in agreement with a median of 2 SLN observed in $>11,000$ patients in the ACOSOG Z0011 trial, the New Start programme and the AMAROS 
Table I. Sensitivity, specificity and predictive values of AUSB based on a threshold of 2 positive nodes for selective axillary preservation for all axillary LN dissections.

\begin{tabular}{lrrr}
\hline AUSB & LN $>2$ & LN $\leq 2$ & Total \\
\hline+ & 67 & 70 & 137 \\
- & 59 & 83 & 142 \\
Total & 126 & 153 & 279 \\
\hline
\end{tabular}

Sensitivity, 0.532 (0.441-0.621); specificity, 0.542 (0.460-0.623) positive predictive value, $0.489(0.430-0.548)$; negative predictive value, 0.584 (0.526-0.640). LN, lymph node; AUSB, axillary ultrasound biopsy.

Table II. Sensitivity, specificity and predictive values of OSNA based on a threshold of 2 positive nodes for selective axillary preservation for all axillae.

\begin{tabular}{lccc}
\hline OSNA & $\mathrm{LN}>2^{\mathrm{a}}$ & $\mathrm{LN} \leq 2^{\mathrm{a}}$ & Total \\
\hline+ & 44 & 58 & 102 \\
- & 10 & 572 & 582 \\
Total & 54 & 630 & 684 \\
\hline
\end{tabular}

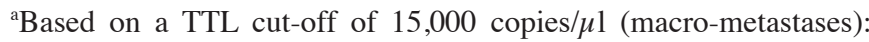
Sensitivity, 0.815 (0.711-0.918); specificity, 0.908 (0.885-0.930); positive predictive value, $0.431(0.335-0.528)$; negative predictive value, 0.983 (0.972-0.992). OSNA, One-Step Nucleic Acid Amplification; TTL, total tumour load; OSNA+, TTL $\geq 15,000$ copies $/ \mu 1$; OSNA-, TTL $<15,000$ copies/ $\mu 1$; LN, lymph node.

Table III. Sensitivity, specificity and predictive values of OSNA based on a threshold of 2 positive nodes for selective axillary preservation for all ACOSOG Z0011 criteria-matched axillae $^{\mathrm{a}}$.

\begin{tabular}{lrrr}
\hline OSNA & $\mathrm{LN}>2^{\mathrm{b}}$ & $\mathrm{LN} \leq 2^{\mathrm{b}}$ & Total \\
\hline+ & 20 & 35 & 55 \\
- & 3 & 441 & 444 \\
Total & 23 & 476 & 499 \\
\hline
\end{tabular}

aPatients are matched to American College of Surgeons Oncology Group Z0011 criteria. 'based on a TTL cut-off of 15,000 copies/ $\mu 1$ (macro-metastases): Sensitivity,0.870(0.732-1.000); specificity, 0.927 (0.903-0.950); positive predictive value, 0.364 (0.237-0.491); negative predictive value, 0.993 (0.986-1.000). TTL, total tumour load; OSNA+, TTL $\geq 15,000$ copies $/ \mu 1$; OSNA-, TTL $<15,000$ copies $/ \mu 1$; LN, lymph node.

trial, collectively $(4,37,38)$. The standard technique of SLNB has an FNR of $<9 \%$ for two nodes (36). However, axillary recurrence risk remains low, even with a range of reported SLNB FNR varying between $6.7-14.8 \%(19,36,39,40)$. OSNA, with an FNR of $1.4 \%$, adds very little to the overall FNR (41).
The NPV for all axillae not associated with breast conserving surgery is 0.949 (95\% CI, 0.906-0.973). This finding suggests that whenever the OSNA TTL is below the cut-off threshold of 15,000 copies $/ \mu 1$, it is likely to be correct in predicting an axillary lymph node burden of $\leq 2$ positive nodes $94.9 \%$ of the time. This patient cohort (that is, those not undergoing breast conserving surgery) includes patients with T3 tumours who are outside the criteria of ACOSOG but within the whole cohort analysis of this study. The FNR of 5\% in this group is also well below the FNR of SLNB for one and two nodes.

Moorman et al (34) reported that only $9.6 \%$ of 1,060 patients (with T1-2 cN0 disease and a maximum of 2 SLNs with macro-metastases) had $>2$ positive axillary nodes on ALND, estimating the risk of having $>2$ positive nodes at $2.2 \%$ for pT1-2. The present study reported a similar low risk of involvement of $>2$ positive nodes of $7.9 \%(54 / 684$ for cT1-3) and 4.6\% (23/499 for cT1-2 cN0) for SLNB + OSNA, using a TTL cut-off of 15,000 copies/ $\mu$ l for predicting $>2$ macro-metastases. The reported rates of isolated axillary recurrence in SLN-disease is $\sim 0.6 \%$ after 3 years and $1.1 \%$ after 5 years (42), similar to the $0.9 \%$ (5 years) and $1.5 \%$ (10 years) for SLN+ disease without ALND in the Z0011 trial $(3,4)$. Thus, with such low estimates of risk, it seems illogical to remain preoccupied with the need to perform AUSB, let alone ALND, on patients with cT1-T2 disease and a clinically negative axilla when local recurrence is $2.5-11 \mathrm{x}$ more likely to occur in a fully irradiated breast than in partially irradiated ipsilateral axilla (3).

SLNB with OSNA, using a TTL cut-off of 15,000 copies/ $\mu 1$, is better than AUSB for assessing nodal involvement in patients naïve to breast surgery and radiotherapy, predicting $\geq 2$ nodal macro-metastases and facilitating the decision to perform ALND or adopt axillary conservation as defined by ACOSOG Z0011 and the National Comprehensive Cancer Network guidelines $(4,43)$. The argument that AUSB+ can avoid unnecessary SLNB for patients requiring ALND is no longer relevant as $51 \%$ of AUSB+ patients had $\leq 2$ positive nodes and were potentially over-treated with ALND. The remaining $49 \%$ of these patients may benefit from ALND, but there is little evidence to support the notion that they all will $(3,44-46)$. Where OSNA is available, the present study recommends that patients who present with cT1-2 cN0 breast carcinoma, should continue to receive AUS as part of their routine follow-up, but only selectively include AUSB if there appear to $\geq 3$ abnormal nodes on imaging. Otherwise, axillary staging is more relevant and practical using SLNB with OSNA. Additionally, for AUSB+ patients, where $\leq 2$ suspicious nodes are seen on AUS, automatic progression to ALND should be challenged by using further intraoperative quantitative assessment with SLNB and OSNA. The decision to proceed with ALND, or adopt axillary conservation, can be determined by OSNA TTL quantification.

In conclusion, where available, OSNA should replace AUSB as the primary and preferred method for axillary assessment in cT1-2 cN0 patients with $\leq 2$ indeterminate nodes on AUS.

\section{Acknowledgements}

Not applicable. 


\section{Funding}

No funding was received.

\section{Availability of data and materials}

The datasets used and/or analysed during the current study are available from the corresponding author on reasonable request. The data may be subject to institutional permission for release.

\section{Authors' contributions}

BI and SK conceived and designed the study, and contributed to data acquisition, analysis and interpretation, and writing and revising the manuscript. VF collected and analysed the OSNA data. NAS was involved in data analysis and interpretation, and in writing and revising the manuscript. SH performed axillary ultrasound examinations, was involved in writing and revising the manuscript, and in acquisition of the ultrasound-guided biopsy data. $\mathrm{OH}$ performed axillary ultrasound examinations and was involved in acquisition of axillary ultrasound biopsy data. PS performed axillary ultrasound examinations and contributed to radiology data acquisition, analysis and interpretation of the data, and in writing and revising the manuscript. GJZ was a major contributor of OSNA data acquisition and interpretation, and in writing and revising the manuscript. NRW contributed to statistical analysis, data interpretation and contributed to writing and revising the manuscript. All authors read and approved the final manuscript.

\section{Ethics approval and consent to participate}

Patient data were anonymised, and collected retrospectively, without influence on patient therapy. This study was approved by the Clinical Effectiveness Unit of the Sheffield Teaching Hospitals Trust (approval no. 7137). Consent for participation from patients was waived.

\section{Patient consent for publication}

Not applicable.

\section{Competing interests}

The authors declare that they have no competing interests.

\section{References}

1. Schipper RJ, van Roozendaal LM, de Vries B, Pijnappel RM, Beets-Tan RG, Lobbes MB and Smidt ML: Axillary ultrasound for preoperative nodal staging in breast cancer patients: Is it of added value? Breast 22: 1108-1113, 2013.

2. Humphrey KL, Saksena MA, Freer PE, Smith BL and Rafferty EA: To do or not to do: Axillary nodal evaluation after ACOSOG Z0011 trial. Radiographics 34: 1807-1816, 2014.

3. Giuliano AE, Ballman K, McCall L, Beitsch P, Whitworth PW, Blumencranz P, Leitch AM, Saha S, Morrow M and Hunt KK: Locoregional recurrence after sentinel lymph node dissection with or without axillary dissection in patients with sentinel lymph node metastases: Long-term follow-up from the american college of surgeons oncology group (Alliance) ACOSOG Z0011 randomized trial. Ann Surg 264: 413-420, 2016.
4. Giuliano AE, Ballman KV, McCall L, Beitsch PD, Brennan MB, Kelemen PR, Ollila DW, Hansen NM, Whitworth PW, Blumencranz PW, et al: Effect of axillary dissection vs. no axillary dissection on 10-year overall survival among women with invasive breast cancer and sentinel node metastasis. The ACOSOG Z0011 (Alliance) randomized clinical trial. JAMA 318: 918-926, 2017.

5. Lee J, Choi JE, Kim SJ, Lee SB, Seong MK, Jeong J, Yoon CS, Kim BK and Sun WY; Korean Breast Cancer Society: Comparative study between sentinel lymph node biopsy and axillary dissection in patients with one or two lymph node metastases. J Breast Cancer 21: 306-314, 2018.

6. Tsujimoto M, Nakabayashi K, Yoshidome K, Kaneko T, Iwase T, Akiyama F, Kato Y, Tsuda H, Ueda S, Sato K, et al: One-step nucleic acid amplification for intraoperative detection of lymph node metastasis in breast cancer patients. Clin Cancer Res 13: 4807-4816, 2007.

7. Di Filippo F, Giannarelli D, Bouteille C, Bernet L, Cano R, Cunnick $\mathrm{G}$ and Sapino A: Elaboration of a nomogram to predict non sentinel node status in breast cancer patients with positive sentinel node, intra-operatively assessed with o nucleic acid amplification method. J Exp Clin Cancer Res 34: 136, 2015.

8. Peg V, Espinosa-Bravo M, Vieites B, Vilardell F, Antúnez JR, de Salas MS, Delgado-Sánchez JJ, Pinto W, Gozalbo F, Petit A, et al: Intraoperative molecular analysis of total tumor load in sentinel lymph node: A new predictor of axillary status in early breast cancer patients. Breast Cancer Res Treat 139: 87-93, 2013.

9. Koca B, Kuru B, Ozen N, Yoruker S and Bek Y: A breast cancer nomogram for prediction of non-sentinel node metastasis-validation of fourteen existing models. Asian Pac J Cancer Prev 15: 1481-1488, 2014.

10. Fung V, Kohlhardt S, Vergani P, Zardin GJ and Williams NR: Intraoperative prediction of the two axillary lymph node macrometastases threshold in patients with breast cancer using a one-step nucleic acid cytokeratin-19 amplification assay. Mol Clin Oncol 7: 755-762, 2017.

11. Peg V, Sansano I, Vieites B, Bernet L, Cano R, Córdoba A, Sancho M, Martín MD, Vilardell F, Cazorla A, et al: Role of total tumour load of sentinel lymph node on survival in early breast cancer patients. Breast 33: 8-13, 2017.

12. Intraoperative tests (RD-100i OSNA system and Metasin test) for detecting sentinel lymph node metastases in breast cancer. NICE diagnostics guidance 8, 2013. Available from: http://www.nice.org.uk/dg8.

13. Raia-Barjat T, Trombert B, Khaddage A, Douchet C, Seffert P, Peoc'h M, Falk AT, Magné N and Chauleur C: OSNA (one-step nucleic acid amplification) sentinel lymph node intraoperative molecular analysis in breast cancer: A cost-benefit analysis. Med Oncol 31: 322, 2014.

14. Brambilla T, Fiamengo B, Tinterri C, Testori A, Grassi MM, Sciarra A, Abbate T, Gatzemeier W, Roncalli $M$ and Di Tommaso L: One-step nucleic acid amplification in breast cancer sentinel lymph node: A single institutional experience and a short review. Front Med (Lausanne) 2: 37, 2015.

15. Stachs A, Thi AT, Dieterich M, Stubert J, Hartmann S, Glass Ä, Reimer T and Gerber B: Assessment of ultrasound features predicting axillary nodal metastasis in breast cancer: The impact of cortical thickness. Ultrasound Int Open 1: E19-E24, 2015.

16. Cho N, Moon WK, Han W, Park IA, Cho J and Noh DY: Preoperative sonographic classification of axillary lymph nodes in patients with breast cancer: Node-to-node correlation with surgical histology and sentinel node biopsy results. AJR Am J Roentgenol 193: 1731-1737, 2009.

17. Bedi DG, Krishnamurthy R, Krishnamurthy S, Edeiken BS, Le-Petross H, Fornage BD, Bassett RL Jr and Hunt KK: Cortical morphologic features of axillary lymph nodes as a predictor of metastasis in breast cancer: In vitro sonographic study. AJR Am J Roentgenol 191: 646-652, 2008.

18. Yang WT, Chang J and Metreweli CL: Patients with breast cancer: Differences in color doppler flow and gray-scale US features of benign and malignant axillary lymph nodes. Radiology 215: 568-573, 2000.

19. Mansel RE, MacNeill F, Horgan K, Goyal A, Britten A, Townson J, Clarke D, Newcombe R, Keshtgar M; Guildford Breast Surgeons, et al: Results of a national training programme in sentinel lymph node biopsy for breast cancer. Br J Surg 100: 654-661, 2013. 
20. NHS Cancer Screening Programmes/Royal College of Pathologists: Pathology Reporting of Breast Disease. NHSBSP publication no. 58, 2005. Available at: https://assets. publishing.service.gov.uk/government/uploads/system/uploads/attachment_data/file/541521/pathology_reporting_of_breast_disease. pdf. Accessed May 21, 2016.

21. Galimberti V, Cole BF, Zurrida S, Viale G, Luini A, Veronesi P, Baratella P, Chifu C, Sargenti M, Intra M, et al: Axillary dissection versus no axillary dissection in patients with sentinel-node micrometastases (IBCSG 23-01): A phase 3 randomised controlled trial. Lancet Oncol 14: 297-305, 2013.

22. Glechner A, Wöckel A, Gartlehner G, Thaler K, Strobelberger M, Griebler U and Kreienberg R: Sentinel lymph node dissection only versus complete axillary lymph node dissection in early invasive breast cancer: A systematic review and meta-analy. Eur J Cancer 49: 812-825, 2013.

23. Straver ME, Meijnen P, van Tienhoven G, van de Velde CJ, Mansel RE, Bogaerts J, Demonty G, Duez N, Cataliotti L, Klinkenbijl J, et al: Role of axillary clearance after a tumor-positive sentinel node in the administration of adjuvant therapy in early breast cancer. J Clin Oncol 28: 731-737, 2010.

24. Caudle AS, Hunt KK, Tucker SL, Hoffman K, Gainer SM, Lucci A, Kuerer HM, Meric-Bernstam F, Shah R, Babiera GV, et al: American college of surgeons oncology group (ACOSOG) Z0011: Impact on surgeon practice patterns. Ann Surg Oncol 19: 3144-3151, 2012.

25. Gainer SM, Hunt KK, Beitsch P, Caudle AS, Mittendorf EA and Lucci A: Changing behavior in clinical practice in response to the ACOSOG Z0011 trial: A survey of the American society of breast surgeons. Ann Surg Oncol 19: 3152-3158, 2012

26. Houssami N, Ciatto S, Turner RM, Cody HS III and Macaskill P. Preoperative ultrasound-guided needle biopsy of axillary nodes in invasive breast cancer: Meta-analysis of its accuracy and utility in staging the axilla. Ann Surg 254: 243-251, 2011.

27. Caudle AS, Kuerer HM, Le-petross HT, Yang W, Yi M, Bedrosian I, Krishnamurthy S, Fornage BD, Hunt KK and Mittendorf EA: Predicting the extent of nodal disease in early-stage breast cancer. Ann Surg Oncol 21: 3440-3447, 2014.

28. Pilewskie M, Mautner SK, Stempel M, Eaton A and Morrow M: Does a positive axillary lymph node needle biopsy result predict the need for an axillary lymph node dissection in clinically node-negative breast cancer patients in the ACOSOG Z0011 era? Ann Surg Oncol 23: 1123-1128, 2016.

29. Ahmed M, Jozsa F, Baker R, Rubio IT, Benson J and Douek M: Meta-analysis of tumour burden in pre-operative axillary ultrasound positive and negative breast cancer patients. Breast Cancer Res Treat 166: 329-336, 2017.

30. Hieken TJ, Trull BC, Boughey JC, Jones KN, Reynolds CA, Shah SS and Glazebrook KN: Preoperative axillary imaging with percutaneous lymph node biopsy is valuable in the contemporary management of patients with breast cancer. Surgery 154 : $831-840,2013$

31. Harris CK, Tran HT, Lee K, Mylander C, Pack D, Rosman M, Tafra L, Umbricht CB, Andrade R, Liang W and Jackson RS: Positive ultrasound-guided lymph node needle biopsy in breast cancer may not mandate axillary lymph node dissection. Ann Surg Oncol 24: 3004-3010, 2017.

32. Stachs A, Göde K, Hartmann S, Stengel B, Nierling U, Dieterich M, Reimer T and Gerber B: Accuracy of axillary ultrasound in preoperative nodal staging of breast cancer-size of metastases as limiting factor. Springerplus 2: 350, 2013.

33. Lee B, Lim AK, Krell J, Satchithananda K, Coombes RC, Lewis JS and Stebbing J: The efficacy of axillary ultrasound in the detection of nodal metastasis in breast cancer. Am J Roentgenol 200: 314-320, 2013.

34. Moorman AM, Bourez RL, Heijmans HJ and Kouwenhoven EA Axillary ultrasonography in breast cancer patients helps in identifying patients preoperatively with limited disease of the axilla. Ann Surg Oncol 21: 2904-2910, 2014.
35. Verheuvel NC, van den Hoven I, Ooms HW, Voogd AC and Roumen RM: The role of ultrasound-guided lymph node biopsy in axillary staging of invasive breast cancer in the post-ACOSOG Z0011 trial era. Ann Surg Oncol 22: 409-415, 2015.

36. Goyal A, Newcombe RG and Mansel RE; Axillary Lymphatic Mapping Against Nodal Axillary Clearance (ALMANAC) Trialists Group: Clinical relevance of multiple sentinel nodes in patients with breast cancer. Br J Surg 92: 438-442, 2005.

37. Goyal A, Newcombe RG, Chhabra A and Mansel RE; ALMANAC Trialists Group: Factors affecting failed localisation and false-negative rates of sentinel node biopsy in breast cancer-results of the ALMANAC validation phase. Breast Cancer Res Treat 99: 203-208, 2006.

38. Donker M, van Tienhoven G, Straver ME, Meijnen P, van de Velde CJ, Mansel RE, Cataliotti L, Westenberg AH, Klinkenbijl JH, Orzalesi L, et al: Radiotherapy or surgery of the axilla after a positive sentinel node in breast cancer (EORTC 10981-22023 AMAROS): A randomised, multicentre, open-label, phase 3 non-inferiority trial. Lancet Oncol 15: 1303-1310, 2014.

39. Krag DN, Anderson SJ, Julian TB, Brown AM, Harlow SP Costantino JP, Ashikaga T, Weaver DL, Mamounas EP, Jalovec LM, et al: Sentinel-lymph-node resection compared with conventional axillary-lymph-node dissection in clinically node-negative patients with breast cancer: Overall survival findings from the NSABP B-32 randomised phase 3 trial. Lancet Oncol 11: 927-933, 2010.

40. Kohrt HE, Olshen RA, Bermas HR, Goodson WH, Wood DJ, Henry S, Rouse RV, Bailey L, Philben VJ, Dirbas FM, et al: New models and online calculator for predicting non-sentinel lymph node status in sentinel lymph node positive breast cancer patients. BMC Cancer 8: 66, 2008.

41. Fujisue M, Nishimura R, Okumura Y, Tashima R, Nishiyama Y, Osako T, Toyozumi Y and Arima N: Clinical significance of CK19 negative breast cancer. Cancers (Basel) 5: 1-11, 2012.

42. Bergkvist L, de Boniface J, Jönsson PE, Ingvar C, Liljegren G and Frisell J; Swedish Society of Breast Surgeons: Axillary recurrence rate after negative sentinel node biopsy in breast cancer: Three year follow-up of the Swedish multicenter cohort study. Ann Surg 247: 150-156, 2008.

43. National Comprehensive Cancer Network. Breast Cancer (Version 4.2020). Available at: https://www.nccn.org/professionals/physician_gls/pdf/breast.pdf. Accessed May 11, 2020

44. International Breast Cancer Study Group, Rudenstam CM, Zahrieh D, Forbes JF, Crivellari D, Holmberg SB, Rey P, Dent D, Campbell I, Bernhard J, et al: Randomized trial comparing axillary clearance versus no axillary clearance in older patients with breast cancer: First results of international breast cancer study group trial 10-93. J Clin Oncol 24: 337-344, 2006.

45. Martelli G, Boracchi P, Ardonino I, Lozza L, Bohm S, Vetrella G and Agresti R: Axillary dissection versus no axillary dissection in older patients with T1N0 breast cancer: 15 -year results of a randomized controlled trial. Ann Surg 256: 920-924, 2012.

46. Agresti R, Martelli G, Sandri M, Tagliabue E, Carcangiu ML, Maugeri I, Pellitteri C, Ferraris C, Capri G, Moliterni A, et al: Axillary lymph node dissection versus no dissection in patients with T1N0 breast cancer: A randomized clinical trial (INT09/98). Cancer 120: 885-893, 2014

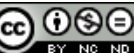

This work is licensed under a Creative Common Attribution-NonCommercial-NoDerivatives 4.0 International (CC BY-NC-ND 4.0) License. 